\title{
Redox Sensitive Nanoparticles with Disulfide Bond Linked Sheddable Shell for Intracellular Drug Delivery
}

\section{Huiyun Wen ${ }^{1,2}$ and Yongyong $\mathrm{Li}^{1 *}$}

${ }^{1}$ Shanghai East Hospital, The Institute for Biomedical Engineering \& Nano Science, Tongji University School of Medicine, Shanghai 200092, P. R. China ${ }^{2}$ School of Chemical Engineering, Northwest University, Xi'an, 710069, P. R. China

\begin{abstract}
Drug delivery systems must overcome a series of biological barriers to escort therapeutical agents to specific pathological site. These include both extracellular barriers and intracellular barriers. To overcome extracellular barriers, the vehicles should have high stability and prolonged circulation time in the blood stream, and sufficient accumulation in disease site. Intracellular barriers are the subsequent barrier that determines successful drug or gene delivery, including effective cellular internalization, endosomal escape, and controllable release. Comprehensive consideration of both extracellular and intracellular barriers to targeted drug and gene delivery is critically important. Nanoparticles (NPs) based drug and gene delivery systems exhibit potential ability to overcome the biological barriers, due to their structures can be tailored to address those barriers such as release the therapeutic agents in the desired site. In this review, we will focus on recent exciting advances in designing redox sensitive nanoparticles for intracellular drug delivery. Those redox sensitive NPs are particularly concentrated on recent emerging NPs with disulfide bond linked sheddable shell like PEG.
\end{abstract}

\section{Graphical Abstract}

Redox sensitive NPs with disulfide linked PEG shell which can respond to tumor intracellular GSH microenvironments for controlled release of therapeutic agents.

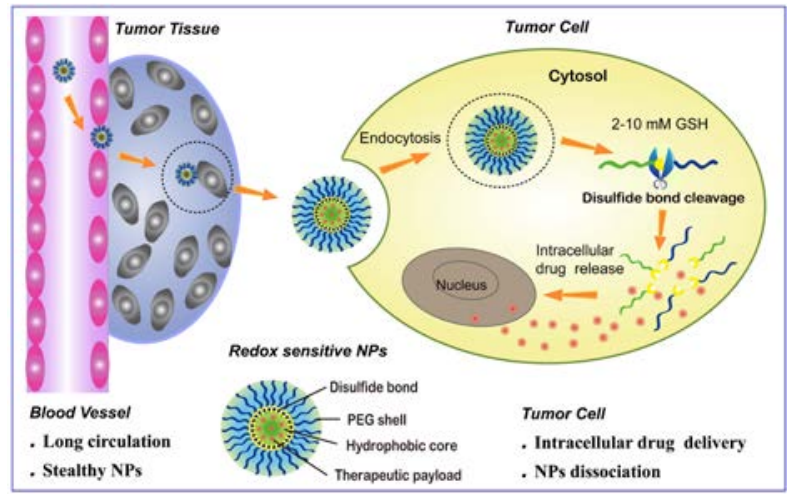

Keywords: Redox sensitive; Nanoparticles; Disulfide bond; Intracellular drug delivery

\section{Introduction}

Advances in nanomedicine and materials chemistry provide tremendous opportunities to develop targeted drug and gene delivery systems in addressing the several therapeutical issues, such as nonspecificity, poor pharmacokinetic profiles, unsatisfactory treatment efficacy, and severe systemic toxicity [1]. To overcome these challenges, drug or gene delivery systems must overcome a series of biological barriers to escort therapeutical agents to specific pathological site. These include both extracellular barriers and intracellular barriers [2]. To overcome extracellular barriers, the vehicles are necessary to have high stability and prolonged circulation time in the blood stream [3]. However, intracellular barrier represents another significant barrier that can hinder drug release, including cellular internalization, endosomal escape, and controllable drug release [4]. Comprehensive consideration of the both extracellular and intracellular barriers to targeted drug and gene delivery is crucial important for the rational design of improved systems.

Nanoparticles (NPs) mediated drug delivery systems have the potential ability to overcome the biological barriers due to EPR effect, efficient drug reservoir, as well as the structural flexibility to be modified to release the therapeutic agents in the desired site [5]. Multiple therapeutic agents including anticancer drug, protein, gene and imaging agents can be carried into nanoparticles physically or chemically [6]. For their encapsulation, a range of morphologically different nanostructures including micelles [7], liposomes [8], polymer-drug conjugate [9], dendrimer [10], hydrogel [11], grapheme oxide [12], silica [13] etc. have been developed (Figure 1). NPs with appropriate size have more opportunities to accumulate at the tumor site through enhanced permeability and retention (EPR) effect [14-16]. Moreover, these NPs can also be conjugated or grafted with affinity

*Corresponding author: Yongyong Li, Shanghai East Hospital, The Institute for Biomedical Engineering \& Nano Science, Tongji University School of Medicine Shanghai 200092, P. R. China, E-mail: yongyong_li@tongji.edu.cn

Received October 28, 2014; Accepted November 24, 2014; Published November 26, 2014

Citation: Wen H, Li Y (2014) Redox Sensitive Nanoparticles with Disulfide Bond Linked Sheddable Shell for Intracellular Drug Delivery. Med chem 4: 748-755. doi:10.4172/2161-0444.1000225

Copyright: (c) 2014 Wen H, et al. This is an open-access article distributed under the terms of the Creative Commons Attribution License, which permits unrestricted use, distribution, and reproduction in any medium, provided the original author and source are credited. 


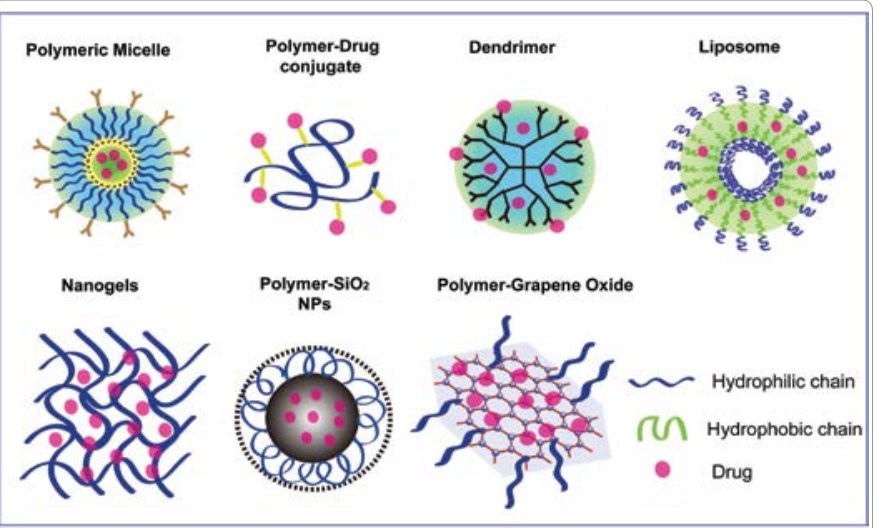

Figure 1: Schematic illustration of representative NPs.

ligands such as folic acid [17], monoclonal antibodies [18,19], peptides [20] or aptamers [21] to increase the selectivity for tumor cells and the tumor microenvironment.

It has been realized that many therapeutics such as anticancer drugs, gene and protein have to be delivered and released in the cellular compartments (e.g. cytoplasm or cell nucleus) so that they can exert therapeutic effects. Thus, NPs engineered with specific triggering mechanism that can release therapeutic agents intracellularly is highly preferred.

In the past several years, development of novel stimuli triggered NPs that release therapeutics in response to tumor intracellular signal, in particular high redox potential, has received great interest. In this review, we will focus on recent exciting advances in designing various redox sensitive NPs related to high glutathione (GSH) level in tumor intracellular microenvironment for intracellular drug delivery. Those redox sensitive NPs are particularly concentrated on recent emerging NPs with disulfide bond linked sheddable shell like PEG. The drug delivery systems engineered with redox sensitive mechanism will open up new possibilities in cancer therapy.

\section{Redox Environment for Intracellular Drug Delivery}

Tumor tissue has different intracellular microenvironments as compared to normal tissue, such as low $\mathrm{pH}$ (4.5-6.5) [22], high temperature $\left(40-42^{\circ} \mathrm{C}\right)$ [23] and over-expressed GSH (2-10 mM) [24]. These biological features can be employed to design NPs with $\mathrm{pH}$, temperature, redox sensitive NPs engineered with intracellular drug release profiles respectively. Particularly, redox-sensitive NPs for drug or gene delivery have received increasing interest over the past years.

GSH is a tripeptide that regulates the cellular reductive microenvironment. There are approximately $2-10 \mathrm{mM}$ GSH levels in intracellular compartments, which are 100-1000 times higher than that in human plasma and blood [25]. Moreover, the cytosolic GSH level in some tumor cells has been found to be at least four times higher than in normal cells [25]. These sharp differences in GSH levels between tumor and normal cells develop the possibility of designing GSH sensitive NPs. NPs with GSH sensitive mechanisms can promote intracellular drug or gene delivery after cellular uptake by disulfide cleavage, and then regulating the intracellular fates of delivered drugs and genes.

\section{Redox Sensitive NPs with Disulfide Bond Linked Sheddable Shell}

Among redox sensitive NPs, those with disulfide-bond-linked sheddable shell is particularly interesting and have attracted broad and intensive attention in the past few years [26,27]. These nanoparticles are generally composed of an inner core with encapsulated therapeutic agents surrounded by hydrophilic, detachable shell [28,29]. The detachable process occurs at a specific manner dependent on the linker between outer shell and inner core. The linker can be redox-, acid-, and enzyme- cleavable according to the aim of the design or condition of delivery process [30]. In this review, it is concentrated on disulfide bond linked nanoparticles. Normally, these nanoparticles keep intact without obvious drug leakage due to structural integrity. In contrast, the shedding of the shell in redox environment via disulfide cleavage would enable the fast drug release intracellularly for structural disassembly of the nanoparticles [25]. Different from conventional stimuli responsive nanoparticles, the structural disassembly of sheddable nanoparticles is fast and complete, since the whole shell can be totally removed from the core exposing drug loaded in the inner core outside. Therefore this strategy can lead to a much more accelerated drug release. Moreover, the drug release is relatively confined into a specific redox area, which is necessary for localized drug delivery. Figure 2 depicts the pathway of these novel nanostructures that act in the biological condition.

For effective therapy, rapid intracellular drug release is highly preferred upon immediate arrival of the delivery system. Therefore, sheddable NPs are particularly suitable for this aim. Most redoxsensitive NPs contain disulfide bond (-S-S-) linked PEG shell, which can be cleaved rapidly in reductive environment analog to tumor intracellular environment, while relatively stable in blood circulation (Figure 2) [26,27,31]. The most often used disulfide bond containing agents are cystamine [32-34], dithiodipropionic acid [35], bis(2-methacryloyloxyethyl) disulfide [36], dimethyl 3,3-dithiobispropionimidate (DTBP) [37], 3,3'-dithiobis(sulfosuccin imidyl propionate) (DTSSP) [38], dithiobis (succinimidylpropionate) (DSP) [39], 2-(pyridyldithio)-ethylamine (PDA) [40], cystamine bisacrylamide (CBA) [41], and N-Succinimidyl 3-(2-pyridyldithio) propionate (SPDP) [42]. Table 1 summarizes some representative NPs that bear disulfide bonds or other crosslinker with a sheddable shell for intracellular drug or gene delivery.

\section{Nano-Formulations with Redox Sensitivity for Intracellular Drug Delivery}

\section{Redox sensitive micelles with disulfide bond linked sheddable shell}

Redox sensitive micelles containing a disulfide linkage between the hydrophilic and hydrophobic segments creates a so-called "shell-

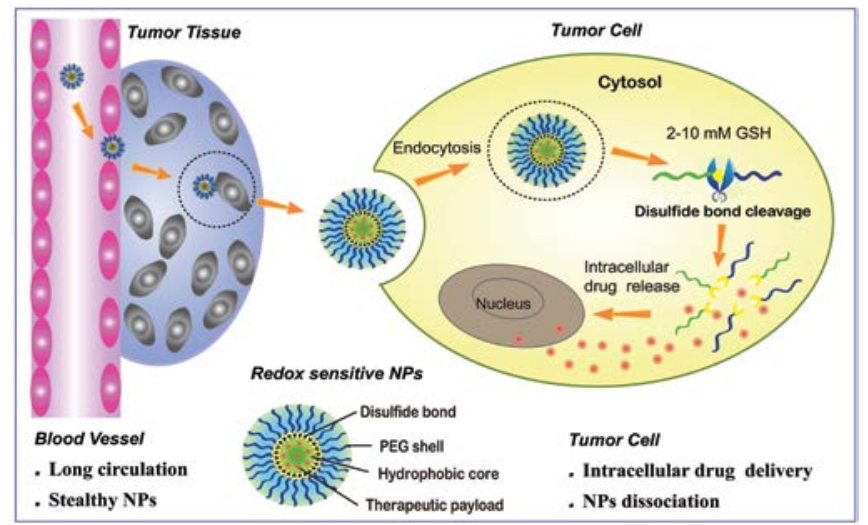

Figure 2: Schematic illustration of redox sensitive NPs with disulfide linked PEG shell which can respond to tumor intracellular GSH microenvironments for controlled release of therapeutic agents. 


\begin{tabular}{|c|c|c|c|c|c|}
\hline NPs & Polymers & Sheddable Shell & Model Drug/Gene & Crosslinker & Ref. \\
\hline \multirow{10}{*}{ Micelle } & PEG-PLys-PPhe & PEG & DTX & DTSSP & [43] \\
\hline & poly(VBPT-co-PEGMA)-S-S-MP & PEG & 6- MP & VBPT & [44] \\
\hline & mPEG-ss-CPP-SA & PEG & Curcumin & DTDP & [45] \\
\hline & PBLG-b-dextran & Dextran & DOX & DTT & [46] \\
\hline & Dextran-g-lipoic acid & Dextran & DOX & DTT & [47] \\
\hline & Dex-SS-PCL & Dextran & DOX & Dex-SS-py+ PCL-SH & [48] \\
\hline & MPEG-SS-PLA & mPEG & PTX & DTT+Py-SS-Py+Py-SH & [49] \\
\hline & PEG-Pu(100\%SS)-PEG & PEG & Pyrene & 2-Hydroxyethyldisulfide & [50] \\
\hline & PEG-SS-COS-SS-PEI & Chitosan & DNA & 3,3'-Dithiodipropionic acid & [51] \\
\hline & PEG-SS-P[Asp(DET)] & PEG & pDNA & Cysteamin & [28] \\
\hline \multirow{4}{*}{$\begin{array}{c}\text { Polymer- } \\
\text { Drug conjugate }\end{array}$} & $(\mathrm{MTX})_{2} \mathrm{PEG}(\mathrm{MTX})_{2}$ & PEG & MTX & Cystamine & [52] \\
\hline & CPT-SS-PEG-SS-CPT & PEG & CPT & Cystamine & [53] \\
\hline & PAsp(-SS-siRNA) & --- & siRNA & PDTA & [54] \\
\hline & multi-siRNA/LPEI & PEI & multi-siRNA & Dithio-bis-maleimidoethane & [55] \\
\hline \multirow{2}{*}{ Dendrimer } & HPHSEP-star-PEPX & --- & DOX & 2-ethoxy-2-oxo- 1,3,2-dioxaphospholane (EP) & [56] \\
\hline & PAMAM-SS-NAC & PAMAM & NAC & SPDP & [57] \\
\hline \multirow{3}{*}{ Graphene Oxide } & NGO-SS-mPEG & mPEG & DXR & Cystamine & [34] \\
\hline & GO-SS-Ce6 & ---- & $\mathrm{Ce} 6$ & Cystamine & [58] \\
\hline & rGO/QC-PEG/Plu-SH & PEG & DOX & Thiol grafted Pluronic & [59] \\
\hline \multirow{3}{*}{$\mathrm{SiO}_{2} \mathrm{NPs}$} & PLGA-Organosilica Silica & --- & Pyrene & TESPDS & [60] \\
\hline & $\beta$-cyclodextrin-MSNs & $\beta-C D$ & Rhodamine B dye & SPDP & [61] \\
\hline & MSNs-SS-mPEG & mPEG & Fluorescein dyes & MSNs-SH+ mPEG-SS-Pyridine & [62] \\
\hline
\end{tabular}

Table 1: Overview of different stealthy NPs engineered with redox sensitive mechanism for drug or gene delivery.

sheddable" micelle [30]. Originally, Kataoka's group [28] developed PEG conjugated cationic micelles [PEG-SS-PAsp(DET)] as nonviral gene vectors. The unique design included a detachable PEG chains upon the intracellular reduction environment (10 mM DTT). Due to PEG detachment, the cationic segment based on poly (aspartamide) could induce more effective endosomal escape with minimal cytotoxicity in the endosome. The micelles showed both a 1-3 orders of magnitude higher pDNA transfection efficiency and a more rapid onset of pDNA expression than that of control micelles containing PEG-PAsp(DET) without disulfide linkage. Building on this earlier work, they examined the in vivo gene expression and antitumor effect of PEG-SS-P[Asp(DET)] micelles with GD4C-TNF- $\alpha$-encoding plasmid by intraperitoneal (i.p.) administration [63]. The results showed that PEG-SS-PAsp(DET) micelles exhibited a higher (Pb0.05) transgene expression compared with PEG-PAsp(DET) in tumors without significant hepatic and renal toxicities.

In a similar strategy, our group has also conducted a series of studies based on PEG-Polylysine (mPEG-PLL) block copolymer [64,65]. We developed mPEG-SS-PLL/DNA complexes with disulfide-linkage for selective and efficient DNA delivery (Figure 3) [66]. These complexes were found to be stable in an aqueous medium with $10 \%$ serum, while fast aggregation occurred in $10 \mathrm{mM}$ GSH environment due to PEG detachment via disulfide bond cleavage. Importantly, mPEG-SS-PLL showed comparable transfection activity and lower cytotoxicity as compared to PEI $25 \mathrm{kDa}$ in both $293 \mathrm{~T}$ and Hela cells, which indicated that the mPEG-SS-PLL complexes was potential non-viral gene vector for gene delivery. Additionally, the above system was also found to be suitable for redox responsive drug delivery [64]. The micelles could rapidly disassemble and release an encapsulated anticancer doxorubicin under tumor-relevant GSH levels, which was three to five times faster than in the absence of GSH.

For a combinational delivery, we recently designed a degradable, sheddable cationic micelles for GSH-mediated intracellular delivery of both drug and gene (Figure 4) [67]. The micelles were about 70 to $200 \mathrm{~nm}$, self-assembled form poly $(\varepsilon$-caprolactone $)-b$-poly $(\mathrm{N}, \mathrm{N}$ dimethylamino-2-ethylmethacrylate) (PCL-SS-PDMA) diblock copolymers. DOX and DNA were chosen as the model drug and gene respectively. The DOX release was remarkably accelerated in $10 \mathrm{mM}$ DTT compared to the control micelles, which is associated with the detachment of the hydrophilic PDMA shell in reductive environment. Meanwhile, the PCL-SS-PDMA block polymers exhibited high DNA binding affinity and cellular uptake efficiency. A ten-fold transfection efficiency of the PCL-SS-PDMA/DNA complexes was found in human cancer cells $\mathrm{KB}$ and CAL-27, as compared to PEI 25K. These novel sheddable and cationic micelles were found to be promising carriers for drug and gene delivery in cancer therapy.

Zhong's group developed reduction sensitive shell-sheddable micelles based on disulfide linked poly(ethylene glycol)-SS-poly( $\varepsilon$ caprolactone) (PEG-SS-PCL) [68] and dextran-SS-PCL (Dex-SS-PCL) [48] copolymers. These prepared micelles showed a faster DOX release profile under $10 \mathrm{mM}$ DTT than that of control micelles without disulfide linkage. Wang's group developed redox sensitive poly( $\varepsilon$-caprolactone)SS-poly(ethyl ethylene phosphate) (PCL-SS-PEEP) micelles for both drug delivery [69] and overcoming multidrug resistance (MDR) [29]. The micelles exhibited GSH-responsive structure change and enhanced intracellular DOX release in A549 cells [69]. Moreover, the redox sensitive micelles were also shown to overcome MDR by enhanced DOX release in MCF-/ADR cells triggered by the high intracellular GSH levels [29]. These results indicate that redox-sensitive micelles exhibit promising advantages in drug delivery as well as improving overall therapeutic efficiency of anticancer drugs.

\section{Redox sensitive prodrugs}

Kataoka et al. [70] pioneered $\mathrm{pH}$ sensitive prodrug design by conjugating DOX onto copolymer chains via an acidic cleavable hydrazone bond. Similarly, redox sensitive prodrugs are fabricated by conjugating therapeutic drugs, protein, or gene onto the backbone of polymers via disulfide linkages, which can release therapeutic agents under reduction environment such as the cytosol or nucleus $(2-10 \mathrm{mM}$ GSH) $[1,71]$.

Our group has covalently attached drug methotrexate (MTX) onto PEG polymers with a disulfide linkage [52]. The prodrug was structured 


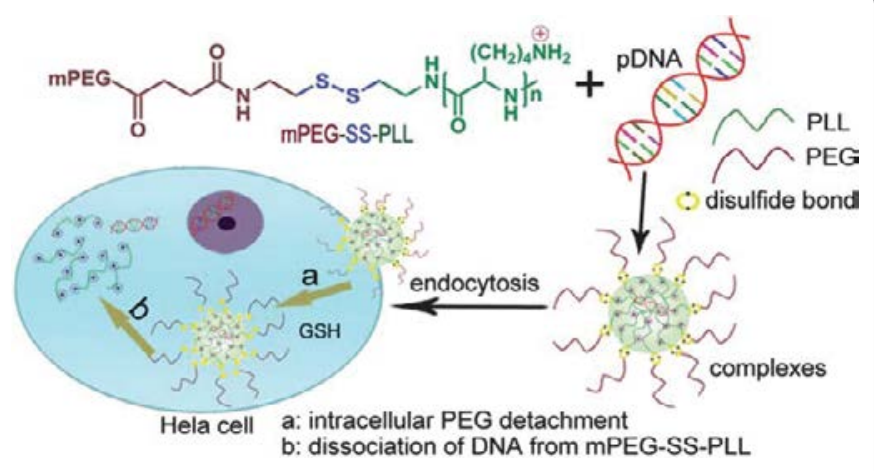

Figure 3: Chemical structure of disulfide-bridged mPEG-SS-PLL block copolymer and schematic illustration of redox-responsive complexes for intracellular DNA delivery [66]. Reprinted with permission from Ref. 66 Copyright 2011 The Royal Society of Chemistry.
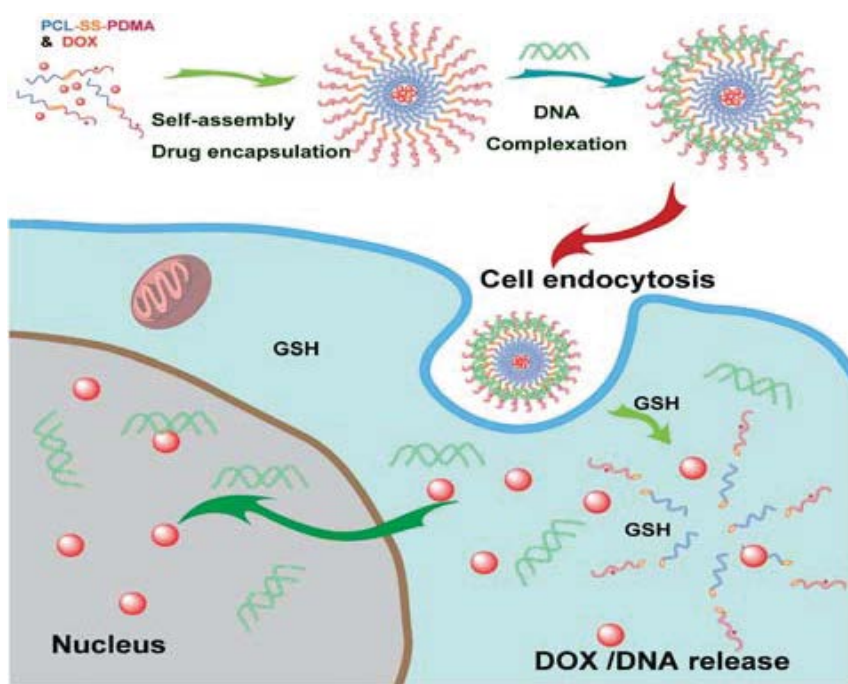

Figure 4: Illustration of redox sensitive PCL-SS-PDMA micelles for intracellular DOX and DNA delivery [67]. Reprinted with permission from Ref. 67. Copyright 2014 The Royal Society of Chemistry.

in H-shape with four arms of MTX drug linked to PEG chain via disulfide linkage (Figure 5). The resulting $\mathrm{H}$-shaped prodrug showed high drug-carrying capability (up to $26 \mathrm{wt} \%$ ) and redox sensitive drug release mechanism. The prodrug self-assembled into NPs bearing a MTX core and exhibited faster drug release rate when exposed to 10 mM DTT. Cell proliferation assays performed with HepG2 cancer cells further demonstrated the pharmacological efficacy of MTX released from prodrug in the presence of elevated GSH.

We also covalently attached camptothecin (CPT) onto PEG chain with a disulfide linkage (CPT-SS-PEG-SS-CPT) [53]. There was a rapid release rate of $4 \%$ per hour during the first $10 \mathrm{~h}$ in the presence of 10 mM DTT. Cell proliferation assays further revealed that the prodrugs effectively decreased HepG2 cell viability, thus demonstrating CPT successfully released from prodrugs engineered with redox sensitive mechanisms. Consequently, the novel prodrug design with disulfide linkage showed great promising in both delivering anti-cancer drugs to desired tumor sites and releasing its cargo in a redox-dependent manner.

It is reported that GSH is abundant in mammalian cells, particularly in drug resistant cells [71-73]. Thus, the redox responsive prodrug was also developed to overcome multidrug resistance (MDR). Liu et al.
[74] developed redox sensitive DOX prodrug (DEX-PEI(-SS-DOX)) by conjugating DOX to DEX-PEI polymers via disulfide linkers. The prodrug self-assembled into micelles with an average size of 100-140 $\mathrm{nm}$ and exhibited rapid drug release under $10 \mathrm{mM}$ DTT. Minimal amount of DOX $(<5 \%)$ was released in the absence of DTT within $48 \mathrm{~h}$, while about 50\% DOX was released within $4 \mathrm{~h}$ in $10 \mathrm{mM}$ DTT. Furthermore, the redox responsive prodrug micelles enhanced the DOX cellular accumulation and achieved endosomal escape in human breast carcinoma multidrug resistan (MCF-7/ADR) cells which could significantly increase the efficiency in overcoming MDR compared to free DOX. These results indicate that redox-sensitive prodrug micelles exhibit promising advantages in overcoming MDR in cancer cells as well as improving overall therapeutic efficiency of anticancer drugs.

\section{Redox sensitive graphene oxide}

Graphene and its derivative graphene oxide (GO) have shown important potential in biological applications owing to their unique physical and chemical properties [75]. In particular, graphene oxide (GO) has shown several advantages especially in drug or gene delivery system [12]. First, due to its large surface area, GO is quite suitable as a loading carrier for various molecules, such as drug and gene, imaging agents [76]. Second, there are some reactive functional groups on its basal plane and edges, such as hydroxyl, epoxide and carboxylic acid, which could be modified or conjugated with polymers to improve its solubility and biocompatibility [77]. Inspired by these important features, numerous reports on GO-based drug or gene delivery have been documented [78,79]. In particular, redox sensitive GO have received great interesting.

Our group developed redox sensitive GO (NGO-SS-mPEG) for tumor selective drug delivery (Figure 6) [34]. The PEG attached on GO sheet via a disulfide linkage and improved the GO stability in blood circulation, while PEG shell detached in reductive environment due to the cleavage of the disulfide linkage, followed by rapid drug (DXR) release. Cell proliferation assays performed with HeLa cells demonstrated the pharmacological efficacy of drug released from NGO-SS-mPEG in high intracellular GSH concentrations. Consistent with the result of cell proliferation assays, confocal laser scanning microscopy (CLSM) results and flow cytomertic analyses further confirmed the enhanced

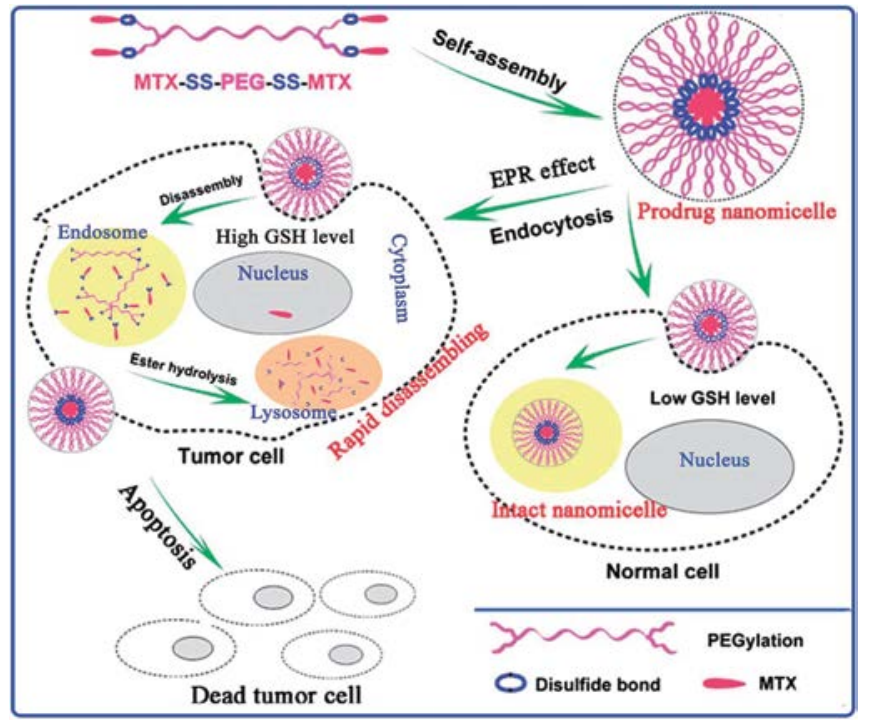

Figure 5: Predicted antitumor activity of redox-sensitive NPs based on H-shaped MTX-SS-PEG-SS-MTX conjugates [52]. Reprinted with permission from Ref. 52. Copyright 2014 The Royal Society of Chemistry. 
DXR release in GSH-OEt pretreated HeLa cells, which associated with the detachment of the PEG shell in reductive environment. Thus, GO composites engineered with GSH-triggered drug release mechanism are able to preferentially deliver encapsulated drug to targeted tumor sites.

GO is not only exploited as a drug carrier, but also a highly efficient energy quencher. Cho et al. [58]. Developed a GO-photosensitizer conjugate (GO-SS-Ce6), in which the photodynamic efficacy of chlorine6 (Ce6) was activated by intracellular GSH. Specifically, Ce6 was conjugated on GO via disulfide linker, and was non-fluorescent and non-phototoxic even upon light irradiation. However, GO-SS-Ce6 exhibited highly fluorescent and phototoxic in A549 cells due to the high GSH triggered cleavage of disulfide bonds, followed by the large amount release of Ce6 from the GO complex. Flow cytometry analysis further confirmed these results. The samples incubating with GO-SSCe6 showed a remarkably improved fluorescence intensity as compared to that of free Ce6 treated A549 cells. Moreover, cell proliferation assays performed with A549 cells demonstrated the photodynamic efficacy of $\mathrm{Ce} 6$ released from GO complexes upon light irradiation in high intracellular GSH concentrations. Notably, these significant findings imply that the GSH responsive GO complex can be a highly efficient and novel approach for photosensitizer delivery.

\section{Redox sensitive silica-based NPs}

Stimulus controlled mesoporous silica for drug or gene delivery is an attractive field, especially since MCM-41 type of mesoporous silica NPs (MSNs) has been proved to be biocompatible [80-82]. Large surface area and ordered mesoporous structure of MSNs is ideal for loading cargo molecules, whereas the functionalization of MSNs surface offers a way to achieve controlled release of loaded molecules [83].

Feng et al. [84] grafted poly(N-acryloxysuccinimide) (PNAS) on the surface of MSNs, followed by cross-linking with cystamine as a "gatekeeper", which could be triggered by redox stimuli for controlled guest molecular delivery in a "switch on/ off" process (Figure 7). The release profile was dependent on the DTT concentration. The loaded rhodamine $\mathrm{B}$ was rapidly released in the presence of a high concentration of DTT $(21.6 \mathrm{mM})$, while a relatively slow delivery at a low DTT concentration $(0.216 \mathrm{mM})$. The loaded rhodamine B released from the MSNs was due to the cleavage of the disulfide linkage on the pore entrance of MSNs in the high reducing DTT environment. In a following study, the authors developed $\beta$-CD-PNAS as the multiresponsive "gatekeeper" of MSNs linked by disulfide bonds [85]. The release profiles exhibited that instantaneous release of loaded calcein was observed in stimuli of adding UV, DTT or a-CD environment,

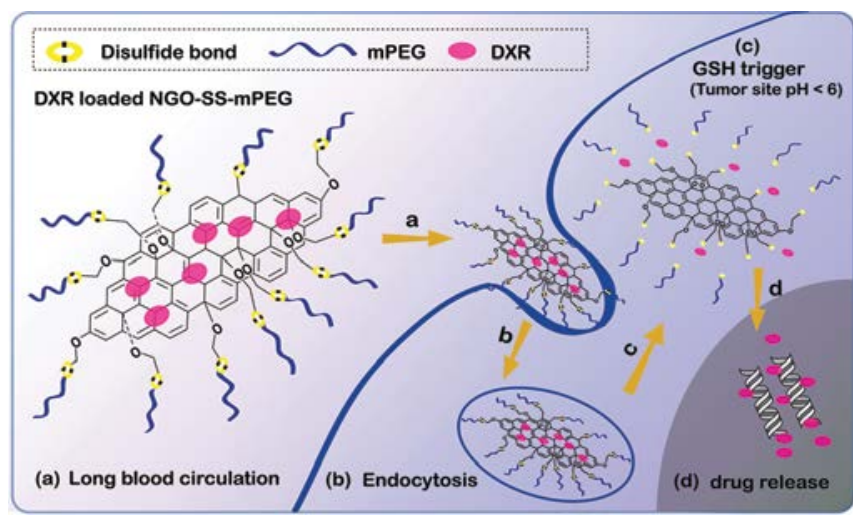

Figure 6: Predicted antitumor activity of redox-sensitive NGO-SS-mPEG [34] Reprinted with permission from Ref. 34. Copyright 2012 John Wiley \& Sons.

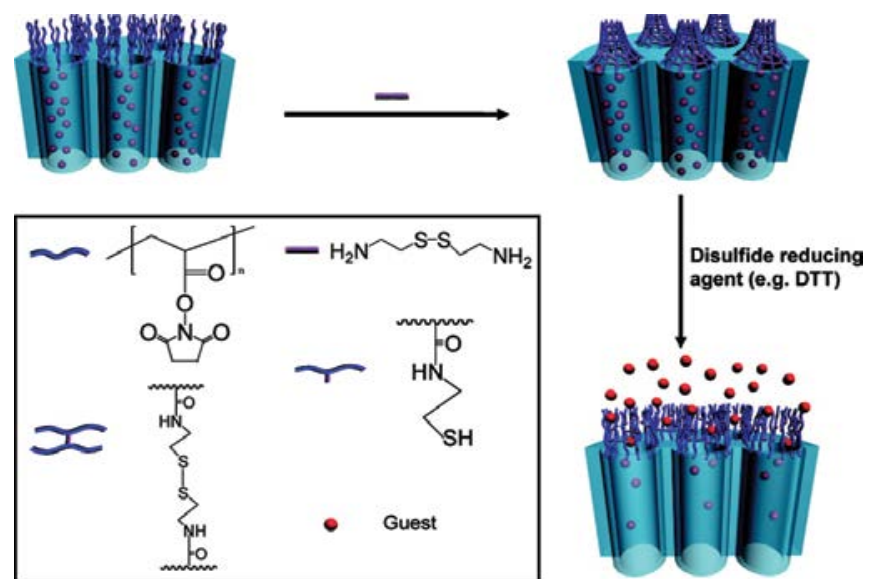

Figure 7: Illustration of redox sensitive PNAS-MSNs for guest molecular delivery [84]. Reprinted with permission from Ref. 86. Copyright 2012 American Chemical Society.

while without external stimuli, release behavior was not obviously observed.

Our group engineered PEG surface-capped MSNs (MSNs-SSmPEG) nanosystem for redox triggered release of fluorescein dye [62]. The PEG shell was anchored on the pore entrance of MSNs via disulfide linker, thus served as gatekeeper to control the on-off behavior of the pores for release of loaded dye. The release behavior investigation demonstrated that the MSNs-SS-mPEG showed a rapid release in the presence of $10 \mathrm{mM} \mathrm{GSH}$ as compared to $0 \mathrm{mM} \mathrm{GSH}$, indicating the accelerated cargo release after the opening of the pores regulated by GSH. CLSM further confirmed the MSNs-SS-mPEG NPs successfully endocytosised into MCF-7 cells and released guest dyes in high intracellular GSH levels.

Kataoka's group designed a smart multilayer assembly (SMA) based on silica for siRNA delivery [86]. The SMA was about $160 \mathrm{~nm}$ in size and was designed in four layers: a polyion complex core for siRNA-loading, a dissolvable silica interlayer for transient core-stabilizing, a polycation interlayer for endosome disrupting, and a detachable PEG shell with redox-sensitivity (Figure 8). The outer PEG shell was connected with PAsp(DET) via disulfide bond (PEG-SS-PAsp(DET)), which could detach from the SMA via the cleavage of disulfide by reducing agents in the endosome/lysosome, followed by the exposure of the PAsp(DET) interlayer for endosome-disrupting. The silica interlayer offered the SMA highly tolerant to dissociation induced by anionic lipids, while siRNA release from the SMA in $24 \mathrm{~h}$ was clearly observed. In contrast with nondisuflide control system, the siRNA released from the SMA within the endosome was significantly lower, suggesting the improved endosomal escape of SMA due to the PEG detachment. Moreover, SMA induced apparent higher gene silencing efficiency in mice tumor tissue without problematic hematological toxicity.

\section{Conclusions and Perspectives}

We have reviewed a number of current designs about redox sensitive NPs especially those containing disulfide bond (-S-S-) linked PEG shell for intracellular drug delivery. The design and development of these redox sensitive NPs linked with disulfide bond for efficiently drug delivery and release represent an alternative of smart approach toward other stimuli sensitive NPs. As evidenced by the presentation of lots of recent designs and studies cited in this review, GSH stimuli have been well-recognized as an ideal internal signal for triggering rapid 

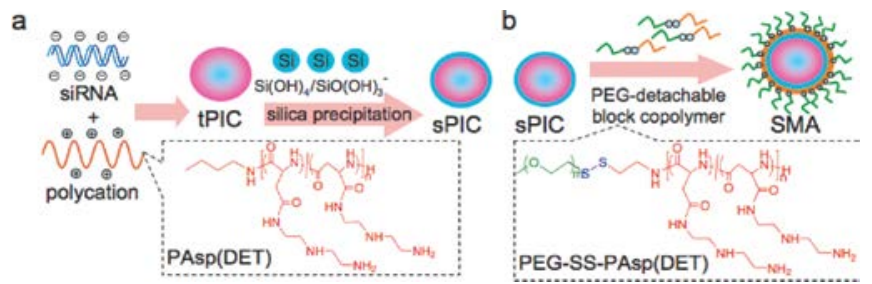

Figure 8: Schemes of a smart multilayered assembly (SMA) based on silica by the layer-by-layer method for siRNA delivery [86]. Reprinted with permission from Ref. 88. Copyright 2012 American Chemical Society.

destabilization of NPs structures in reductive environment analog to tumor intracellular environment, which will release drug rapidly inside cells and significantly enhance drug efficacy. Moreover, the past several years have seen rapid progress in the design of various redox sensitive NPs for overcoming the biological barriers in drug or gene delivery which can uniquely resolve the stability dilemma of NPs. In this review, the design of sheddable NPs sensitive to intracellular reductive tumor conditions may represent an attractive strategy for intracellular drug and gene delivery.

However, a vast variety of redox sensitive NPs have been reported, only a small number of them has been tested in preclinical, in vivo models. It should further be noted that lot of the coating polymers are not biodegradable or biocompatible, which are not suitable for drug or gene delivery in clinical use. Thus, more efforts need to promote studies in the reasonable design of redox sensitive NPs.

As we have reviewed, both the understanding of biological barrier and the merits of intracellular drug or gene delivery led to the successful design of redox sensitive NPs. Looking to the future, we expect that our review will further aid researchers in developing NPs that are capable of reaching the clinical setting.

\section{Acknowledgements}

We gratefully acknowledge financial support from National Natural Science Foundation of China (51173136, 21306152 and 21104059), Shanghai RisingStar Program (12QA1403400), and the Natural Science Basic Research Plan in Shaanxi Province of China (2014JQ2067 and14JK1744).

\section{References}

1. Ge Z, Liu S (2013) Functional block copolymer assemblies responsive to tumor and intracellular microenvironments for site-specific drug delivery and enhanced imaging performance. Chem Soc Rev 42: 7289-7325.

2. Li L, Sun J, He Z (2013) Deep penetration of nanoparticulate drug delivery systems into tumors: challenges and solutions. Curr Med Chem 20: 2881-2891.

3. Elsabahy M, Wooley KL (2012) Design of polymeric nanoparticles for biomedical delivery applications. Chem Soc Rev 41: 2545-2561.

4. Nie $S$ (2010) Understanding and overcoming major barriers in cancer nanomedicine. Nanomedicine (Lond) 5: 523-528.

5. Cheng R, Meng F, Deng C, Klok HA, Zhong Z (2013) Dual and multi-stimul responsive polymeric nanoparticles for programmed site-specific drug delivery. Biomaterials 34: 3647-3657.

6. Jabr-Milane L, van Vlerken L, Devalapally $\mathrm{H}$, Shenoy D, Komareddy S, et al. (2008) Multi-functional nanocarriers for targeted delivery of drugs and genes. $J$ Control Release 130: 121-128.

7. Kataoka K, Harada A, Nagasaki Y (2001) Block copolymer micelles for drug delivery: design, characterization and biological significance. Adv Drug Deliv Rev 47: 113-131.

8. Opanasopit P, Tragulpakseerojn J, Apirakaramwong A, Ngawhirunpat T, Rojanarata T, et al. (2011) The development of poly-L-arginine-coated liposomes for gene delivery. Int J Nanomedicine 6: 2245-2252.

9. John JV, Johnson RP, Heo MS, Moon BK, Byeon SJ, et al. (2015) Polymer-
Block-Polypeptides and Polymer-Conjugated Hybrid Materials as Stimuli-Responsive Nanocarriers for Biomedical Applications. J Biomed Nanotechnol 1: 1-39.

10. Pan D, She W, Guo C, Luo K, Yi Q, et al. (2014) PEGylated dendritic diaminocyclohexyl-platinum (II) conjugates aspH-responsive drug delivery vehicles with enhanced tumor accumulation and antitumor efficacy. Biomaterials 35: 10080-10092.

11. Chacko RT, Ventura J, Zhuang J, Thayumanavan S (2012) Polymer nanogels: a versatile nanoscopic drug delivery platform. Adv Drug Deliv Rev 64: 836-851.

12. Pan Y, Sahoo NG, Li L (2012) The application of graphene oxide in drug delivery. Expert Opin Drug Deliv 9: 1365-1376.

13. Gouda N, Miyata K, Christie RJ, Suma T, Kishimura A, et al. (2013) Silica nanogelling of environment-responsive PEGylated polyplexes for enhanced stability and intracellular delivery of siRNA. Biomaterials 34: 562-570.

14. Park JH, Lee S, Kim JH, Park K, Kim K, et al. (2008) Polymeric nanomedicine for cancer therapy. Prog Polym Sci 33: 113-137.

15. Maeda H, Nakamura H, Fang J (2013) The EPR effect for macromolecular drug delivery to solid tumors: Improvement of tumor uptake, lowering of systemic toxicity, and distinct tumor imaging in vivo. Adv Drug Deliv Rev 65: 71-79.

16. Maeda H, Bharate GY, Daruwalla J (2009) Polymeric drugs for efficient tumortargeted drug delivery based on EPR-effect. Eur J Pharm Biopharm 71: 409-419.

17. Low PS, Henne WA, Doorneweerd DD (2008) Discovery and development of folic-acid-based receptor targeting for imaging and therapy of cancer and inflammatory diseases. Acc Chem Res 41: 120-129.

18. Qian CC, Wang Y, Chen YT, Zeng LJ, Zhang QB, et al. (2013) Suppression of pancreatic tumor growth by targeted arsenic delivery with anti-CD44v6 single chain antibody conjugated nanoparticles. Biomaterials 34: 6175-6184.

19. Han H, Davis ME (2013) Single-antibody, targeted nanoparticle delivery of camptothecin. Mol Pharm 10: 2558-2567.

20. Quan CY, Chen JX, Wang HY, Li C, Chang C, et al. (2010) Core-Shell Nanosized Assemblies Mediated by the alpha-beta Cyclodextrin Dimer with a Tumor-Triggered Targeting Property. Acs Nano 4: 4211-4219.

21. Farokhzad OC, Jon SY, Khademhosseini A, Tran TNT, LaVan DA, et al. (2004) Nanopartide-aptamer bioconjugates: A new approach for targeting prostate cancer cells. Cancer Research 64: 7668-7672.

22. Gao GH, Li Y, Lee DS (2013) Environmental pH-sensitive polymeric micelles for cancer diagnosis and targeted therapy. J Control Release 169: 180-184.

23. Pradhan P, Giri J, Rieken F, Koch C, Mykhaylyk O, et al. (2010) Targeted temperature sensitive magnetic liposomes for thermo-chemotherapy. J Control Release 142: 108-121.

24. Cheng R, Feng F, Meng F, Deng C, Feijen J, et al. (2011) Glutathione-responsive nano-vehicles as a promising platform for targeted intracellular drug and gene delivery. J Control Release 152: 2-12.

25. Meng F, Hennink WE, Zhong Z (2009) Reduction-sensitive polymers and bioconjugates for biomedical applications. Biomaterials 30: 2180-2198.

26. Li CG, Zhao S, Li JJ, Yin, YJ (2013) Polymeric Biomaterials Containing Thiol/ Disulfide Bonds. Prog Chem 25: 122-134.

27. Bauhuber S, Hozsa C, Breunig M, Gopferich A (2009) Delivery of nucleic acids via disulfide-based carrier systems. Adv Mater 21: 3286-3306.

28. Takae S, Miyata K, Oba M, Ishii T, Nishiyama N, et al. (2008) PEG-detachable polyplex micelles based on disulfide-linked block catiomers as bioresponsive nonviral gene vectors. J Am Chem Soc 130: 6001-6009.

29. Wang YC, Wang F, Sun TM, Wang J (2011) Redox-Responsive Nanoparticles from the Single Disulfide Bond-Bridged Block Copolymer as Drug Carriers for Overcoming Multidrug Resistance in Cancer Cells. Bioconjugate Chem 22: 1939-1945.

30. Romberg B, Hennink WE, Storm G (2008) Sheddable coatings for long-circulating nanoparticles. Pharm Res 25: 55-71.

31. Saito G, Swanson JA, Lee KD (2003) Drug delivery strategy utilizing conjugation via reversible disulfide linkages: role and site of cellular reducing activities. Adv Drug Deliv Rev 55: 199-215.

32. Li YT, Smith AE, Lokitz BS, McCormick CL (2007) In situ formation of gold"decorated" vesicles from a RAFT-synthesized, thermally responsive block copolymer. Macromolecules 40: 8524-8526. 
33. Cai XJ, Dong CY, Dong HQ, Wang GM, Pauletti GM, et al. (2012). Effective Gene Delivery Using Stimulus-Responsive Catiomer Designed with RedoxSensitive Disulfide and Acid-Labile Imine Linkers Biomacromolecules 13: 1024-1034.

34. Wen H, Dong C, Dong H, Shen A, Xia W, et al. (2012) Engineered redoxresponsive PEG detachment mechanism in PEGylated nano-graphene oxide for intracellular drug delivery. Small 8: 760-769.

35. Huang Q, Liu T, Bao CY, Lin QN, Ma MX, et al. (2014) Light and reductive dual stimuli-responsive PEI nanoparticles: "AND" logic response and controllable release. J Mater Chem 2: 3333-3339.

36. Li Y, Xiao K2, Zhu W3, Deng W2, Lam KS4 (2014) Stimuli-responsive crosslinked micelles for on-demand drug delivery against cancers. Adv Drug Deliv Rev 66: 58-73.

37. Oe Y, Christie RJ, Naito M, Low SA, Fukushima S, et al. (2014) Actively-targeted polyion complex micelles stabilized by cholesterol and disulfide cross-linking for systemic delivery of siRNA to solid tumors. Biomaterials 35: 7887-7895.

38. Koo AN, Min KH, Lee HJ, Lee SU, Kim K, et al. (2012) Tumor accumulation and antitumor efficacy of docetaxel-loaded core-shell-corona micelles with shellspecific redox-responsive cross-links. Biomaterials 33: 1489-1499.

39. Feng M, Ibrahim BM, Wilson EM, Doh KO, Bergman BK, et al. (2014) Stabilization of a hyaluronate-associated gene delivery system using calcium ions. Biomater Sci-Uk 2: 936-942.

40. Novo L, Mastrobattista E, van Nostrum CF, Hennink WE (2014) Targeted decationized polyplexes for cell specific gene delivery. Bioconjug Chem 25: $802-$ 812

41. Wang LH, Xu XM, Hong CY, Wu DC, Yu ZQ, et al. (2014) Biodegradable large compound vesicles with controlled size prepared via the self-assembly of branched polymers in nanodroplet templates. Chem Commun (Camb) 50 : 9676-9678.

42. Wang C, Chen B2, Zou M3, Cheng G4 (2014) Cyclic RGD-modified chitosan/ graphene oxide polymers for drug delivery and cellular imaging. Colloids Surf B Biointerfaces 122: 332-340.

43. Koo AN, Min KH, Lee HJ, Lee SU, Kim K, et al. (2012) Tumor accumulation and antitumor efficacy of docetaxel-loaded core-shell-corona micelles with shellspecific redox-responsive cross-links. Biomaterials 33: 1489-1499.

44. Zhuang Y, Su Y, Peng Y, Wang D, Deng H, et al. (2014) Facile fabrication of redox-responsive thiol-containing drug delivery system via RAFT polymerization. Biomacromolecules 15: 1408-1418.

45. Wang J, Yang G, Guo X, Tang Z, Zhong Z2, et al. (2014) Redox-responsive polyanhydride micelles for cancer therapy. Biomaterials 35: 3080-3090.

46. Zhang A, Zhang Z, Shi F, Xiao C, Ding J, et al. (2013) Redox-sensitive shellcrosslinked polypeptide-block-polysaccharide micelles for efficient intracellular anticancer drug delivery. Macromol Biosci 13: 1249-1258.

47. Li YL, Zhu L, Liu Z, Cheng R, Meng F, et al. (2009) Reversibly stabilized multifunctional dextran nanoparticles efficiently deliver doxorubicin into the nuclei of cancer cells. Angew Chem Int Ed Engl 48: 9914-9918.

48. Sun H, Guo B, Li X, Cheng R, Meng F, et al. (2010) Shell-sheddable micelles based on dextran-SS-poly(epsilon-caprolactone) diblock copolymer for efficient intracellular release of doxorubicin. Biomacromolecules 1: 848-854.

49. Song N, Liu W, Tu Q, Liu R, Zhang Y, et al. (2011) Preparation and in vitro properties of redox-responsive polymeric nanoparticles for paclitaxel delivery. Colloids Surf B Biointerfaces 87: 454-463.

50. Tong R, Xia HS, Lu XL (2013) Fast release behavior of block copolymer micelles under high intensity focused ultrasound/redox combined stimulus. J Mat Chem B 1: 886-894.

51. Jia L, Li Z, Zhang D, Zhang Q, Shen J, et al. (2013) Redox-responsive catiomer based on PEG-ss-chitosan oligosaccharide-ss-polyethylenimine copolymer for effective gene delivery. Polym Chem 4: 156.

52. Dong H, Dong C, Xia W, Li Y, Ren T (2014) Self-assembled, redox-sensitive $\mathrm{H}$-shaped pegylated methotrexate conjugates with high drug-carrying capability for intracellular drug delivery. Med Chem Comm 5: 147.

53. Li XQ, Wen HY, Dong HQ, Xue WM, Pauletti GM, et al. (2011) Self-assembling nanomicelles of a novel camptothecin prodrug engineered with a redox-responsive release mechanism. Chem Commun 47: 8647-8649.

54. Takemoto H, Ishii A, Miyata K, Nakanishi M, Oba M, et al. (2010) Polyion complex stability and gene silencing efficiency with a siRNA-grafted polymer deliv- ery system. Biomaterials 31: 8097-8105.

55. Mok H, Lee SH, Park JW, Park TG (2010) Multimeric small interfering ribonucleic acid for highly efficient sequence-specific gene silencing. Nat Mater 9: 272-278.

56. Liu J, Pang Y, Huang W, Zhu Z, Zhu X, et al. (2011) Redox-responsive polyphosphate nanosized assemblies: a smart drug delivery platform for cancer therapy. Biomacromolecules 12: 2407-2415.

57. Kannan -dendrimer bioconjugate NAC.pdf

58. Cho Y, Choi Y (2012) Graphene oxide-photosensitizer conjugate as a redoxresponsive theranostic agent. Chem Commun (Camb) 48: 9912-9914.

59. Al-Nahain A, Lee SY, In I, Lee KD, Park SY (2013) Triggered pH/redox re sponsive release of doxorubicin from prepared highly stable graphene with thiol grafted Pluronic. Int J Pharm 450: 208-217.

60. Quesada M, Muniesa C, Botella P (2013) Hybrid PLGA-Organosilica Nanoparticles with Redox-Sensitive Molecular Gates. Chemistry of Materials 25: 2597 2602.

61. Nadrah P, Maver U, Jemec A, Tiß̊ $\AA_{j}$ ler T, Bele M, et al. (2013) Hindered disulfide bonds to regulate release rate of model drug from mesoporous silica. ACS App Mater Interfaces 5: 3908-3915.

62. Cui $Y$, Dong H Cai X, Wang D, Li Y (2012) Mesoporous silica nanoparticles capped with disulfide-linked PEG gatekeepers for glutathione-mediated controlled release. ACS Appl Mater Interfaces 4: 3177-3183.

63. Kumagai M, Shimoda S, Wakabayashi R, Kunisawa Y, Ishii T, et al. (2012 Effective transgene expression without toxicity by intraperitoneal administration of PEG-detachable polyplex micelles in mice with peritoneal dissemination. Control Release 160: 542-551.

64. Wen HY, Dong HQ, Xie WJ, Li YY, Wang K, et al. (2011) Rapidly disassembling nanomicelles with disulfide-linked PEG shells for glutathione-mediated intracellular drug delivery. Chem Commun (Camb) 47: 3550-3552.

65. Ren TB, Xia WJ, Dong HQ, Li YY (2011) Sheddable micelles based on disulfide-linked hybrid PEG-polypeptide copolymer for intracellular drug delivery. Polymer 52: 3580-3586.

66. Cai XJ, Dong HQ, Xia WJ, Wen HY, Li XQ, et al. (2011) Glutathione-mediated shedding of PEG layers based on disulfide-linked catiomers for DNA delivery. J Mater Chem 2: 14639.

67. Li Y, Lei X, Dong H, Ren T (2014) Sheddable, degradable, cationic micelles enabling drug and gene delivery. RSC Advances 4: 8165

68. Sun HL, Guo BN, Cheng R, Meng FH, Liu HY, et al. (2009) Biodegradable micelles with sheddable poly(ethylene glycol) shells for triggered intracellular release of doxorubicin. Biomaterials 30: 6358-6366.

69. Tang LY, Wang YC, Li Y, Du JZ, Wang J (2009) Shell-detachable micelles based on disulfide-linked block copolymer as potential carrier for intracellular drug delivery. Bioconjug Chem 20: 1095-1099.

70. Bae Y, Fukushima S, Harada A, Kataoka K (2003) Design of environmentsensitive supramolecular assemblies for intracellular drug delivery: polymeric micelles that are responsive to intracellular $\mathrm{pH}$ change. Angew Chem Int Ed Engl 42: 4640-4643.

71. Wang J, Sun X, Mao W, Sun W, Tang J, et al. (2013) Tumor redox heterogeneity-responsive prodrug nanocapsules for cancer chemotherapy. Adv Mater 25: 3670-3676.

72. Lee FY, Vessey A, Rofstad E, Siemann DW, Sutherland RM (1989) Heterogeneity of glutathione content in human ovarian cancer. Cancer Res 49: 5244 5248

73. Wang J, Sun X, Mao W, Sun W, Tang J, et al. (2013) Tumor redox heterogeneity-responsive prodrug nanocapsules for cancer chemotherapy. Adv Mater 25: 3670-3676.

74. Liu P, Shi B, Yue C, Gao G, Li P, et al. (2013) Dextran-based redox-responsive doxorubicin prodrug micelles for overcoming multidrug resistance. Polym Chem 4: 5793

75. Feng L, Liu Z (2011) Graphene in biomedicine: opportunities and challenges. Nanomedicine (Lond) 6: 317-324.

76. Liu Z, Robinson JT, Sun X, Dai H (2008) PEGylated nanographene oxide for delivery of water-insoluble cancer drugs. J Am Chem Soc 130: 10876-10877.

77. Georgakilas V, Otyepka M, Bourlinos AB, Chandra V, Kim N, et al. (2012) 
Citation: Wen H, Li Y (2014) Redox Sensitive Nanoparticles with Disulfide Bond Linked Sheddable Shell for Intracellular Drug Delivery. Med chem 4: 748-754. doi:10.4172/2161-0444.1000225

Functionalization of graphene: covalent and non-covalent approaches, derivatives and applications. Chem Rev 112: 6156-6214.

78. Zhang L, Lu Z, Zhao Q, Huang J, Shen H, et al. (2011) Enhanced chemotherapy efficacy by sequential delivery of siRNA and anticancer drugs using PEl-grafted graphene oxide. Small, 7: 460-464.

79. Yang K, Wan J, Zhang S, Zhang Y, Lee ST, et al. (2011) In vivo pharmacokinetics, long-term biodistribution, and toxicology of PEGylated graphene in mice. ACS Nano 5: 516-522.

80. Chen Y, Chen H, Shi J (2014) Drug delivery/imaging multifunctionality of mesoporous silica-based composite nanostructures. Expert Opin Drug Deliv 11 917-930.

81. Slowing II, Vivero-Escoto JL, Wu CW, Lin VS (2008) Mesoporous silica nanoparticles as controlled release drug delivery and gene transfection carriers. Adv Drug Deliv Rev 60: 1278-1288.
82. Vivero-Escoto JL, Slowing II, Trewyn BG, Lin VS (2010) Mesoporous silica nanoparticles for intracellular controlled drug delivery. Small 6: 1952-1967.

83. Zhao Y, Vivero-Escoto JL, Slowing II, Trewyn BG, Lin VS (2010) Capped mesoporous silica nanoparticles as stimuli-responsive controlled release systems for intracellular drug/gene delivery. Expert Opin Drug Deliv 7: 1013-1029.

84. Liu R, Zhao X, Wu T, Feng P (2008) Tunable redox-responsive hybrid nanogated ensembles. J Am Chem Soc 130: 14418-14419.

85. Liu R, Zhang Y, Feng P (2009) Multiresponsive supramolecular nanogated ensembles. J Am Chem Soc 131: 15128-15129.

86. Suma T, Miyata K, Anraku Y, Watanabe S, Christie RJ, et al. (2012) Smart multilayered assembly for biocompatible siRNA delivery featuring dissolvable silica, endosome-disrupting polycation, and detachable PEG. Acs Nano 6: 6693-6705. 